Mathematical Modelling and Analysis

Volume 21 Number 6, November 2016, 852-868

http://dx.doi.org/10.3846/13926292.2016.1248510

(c) Vilnius Gediminas Technical University, 2016
Publisher: Taylor\&Francis and VGTU

http://www.tandfonline.com/TMMA

ISSN: $1392-6292$

eISSN: $1648-3510$

\title{
Torsional Dynamic Response of a Carbon Nanotube Embedded in Visco-Pasternak's Medium
}

\author{
Ashraf M. Zenkour ${ }^{a, b}$ \\ ${ }^{a}$ Department of Mathematics, Faculty of Science, King Abdulaziz University \\ P.O. Box 80203, Jeddah 21589, Saudi Arabia \\ ${ }^{b}$ Department of Mathematics, Faculty of Science, Kafrelsheikh University \\ Kafrelsheikh 33516, Egypt \\ E-mail(corresp.): zenkour@kau.edu.sa \\ E-mail: zenkour@sci.kfs.edu.eg
}

Received January 25, 2016; revised October 11, 2016; published online November 15, 2016

\begin{abstract}
The torsional dynamics of carbon nanotubes embedded in viscoelastic medium are presented by using the nonlocal elasticity theory. The medium is considered as a foundation model which characterized by the linear Winkler's modulus, Pasternak's (shear) foundation modulus and the damping coefficient. The governing torsional equation is obtained and solved for nanotubes subjected to various boundary conditions and stated under different loads. The effects of some parameters like nonlocal parameter, nanotube length, Winkler's modulus, and damping coefficient on the angular displacement of the nanotube are investigated in detail. The angular displacements are very sensitive to all parameters, especially the inclusion of the viscous damping foundation. Present results can be useful in design of future nano composites, nano electromechanical systems like nano position sensors and linear servomotors. Sample angular displacements are tabulated and plotted for sensing the effect of all used parameters and to investigate the visco-Pasternak's parameters for future comparisons.
\end{abstract}

Keywords: nonlocal elasticity theory, nanotube, angular displacement, visco-Pasternak's medium.

AMS Subject Classification: $74 \mathrm{Fxx}$.

\section{Introduction}

In 1991, Iijima [16] has discovered some types of very thin and may be long cylinders made of carbon which are said to be carbon nanotubes (CNTs). Carbon nanotubes have extraordinary physical properties like high mechanical strength, good electrical conductivity, and wide range of possible uses [1,23]. They are the ideal reinforcement for high performance composites due to their small size, low density, high stiffness and high strength; and therefore, they are 
considered as new generation of reinforcing phase in fabricating nano-composite materials. With these superior properties, CNTs are used by scientists in several areas like sensor technologies, composites and electromechanical systems. Most types of the CNTs are cylindrical macromolecules composed of a highly ordered sheet of carbon atoms in a periodic hexagonal arrangement rolled into a tube. For the modeling of and treating with the CNTs we have used two models (the molecular dynamic $[2,21,30]$ and the continuum mechanics $[7,11,12,13,17,19])$ as well as the experimental methods. We can deal with the molecular dynamic simulation if the structures have small scale like the CNTs and the process is made in a short time interval. The continuum model can also be used as an alternative, although it is not size-dependent.

In most applications, we have noticed that the CNTs are embedded in an elastic foundation medium. The first type of elastic foundation is presented by Winkler as a one-parameter elastic foundation. It is used in a lot of bending, vibration, and torsional buckling of CNTs problems [14,15,22, 25, 28]. For instance, Han and Lu [14] have examined the torsional buckling of a doublewalled CNT embedded in an elastic medium. The effects of surrounding elastic medium and van der Waals forces between the inner and outer nanotubes are taken into consideration. Sun and Liu [25] have investigated the combined torsional buckling of multi-walled CNTs under combined torque, axial loading and radial pressures based on the continuum mechanics model taking into account the effect of the van der Waals interaction between adjacent tubes. Natsuki et al. [22] have studied the influences of the aspect ratio, the buckling modes, and the surrounding elastic medium on the torsional instability of double-walled CNTs. Hao et al. [15] have presented the small scale effect on torsional buckling of multi-walled CNTs.

Winkler's model cannot be taken into account as an exact approximation of the elastic foundation since it considers only the parameter representing the normal pressure. So, Pasternak has presented the second type of elastic foundation since he has considered both normal pressure and transverse shear stress to be a more generalized depiction of the elastic foundation. A limited number of studies have used the two-parameter Pasternak's model for depicting the mechanical characteristics of the elastic foundation. Murmu and Pardhan [20] have presented the two-parameter Winkler-Pasternak model as well as the nonlocal elasticity theory for the buckling analysis of single-walled CNTs. Mohammadimehr et al. [18] have investigated the effects of the two-parameter surrounding elastic foundation for the torsional buckling of a double-walled CNT. Arani et al. [5] have studied the thermal effect on the buckling analysis of a double-walled CNT embedded in an elastic medium and modeled the interaction between matrix and the outer tube as a Pasternak's foundation. Extensive studies have been conducted on the mechanical properties of CNTs embedded in elastic foundation such as static bending [27], free vibration and dynamic response [29], and buckling [26].

Physical environments like fluid flow or biological tissue can be modelled as a viscoelastic medium, which has damping and elastic characteristics, and the effect to the torsional vibration of CNT is a very interesting topic for researchers. In general, CNTs can be modelled using continuum theories. Unlike 
from classical theory, Eringen's nonlocal elasticity theory includes size effects. Eringen $[8,9]$ stated that: "The stress at a point is a functional of the strain field at every point of the continuum". Especially in nano dimensions, size dependency gains much importance because of atomic interactions. Zhen et al. [33] have studied the transverse vibration of fluid-conveying double walled CNTs embedded in biological soft tissue. Soltani et al. [24] studied the vibration of a viscous-fluid-conveying double-walled CNT embedded in visco-elastic medium. Arani and Roudbari [4] have developed the nonlocal longitudinal and transverse vibrations of coupled boron nitride nanotube system under a moving nanoparticle using piezoelastic theory and surface stress based on EulerBernoulli beam. Arani et al. [3] have studied the nonlinear free vibration and instability of fluid-conveying double-walled boron nitride nanotubes embedded in viscoelastic medium.

The torsional dynamic response problem of a carbon nanotube embedded in a visco-Pasternak's medium displays various characters, and the solution becomes difficult (Zenkour [31,32]). The present paper deals with the dynamic response of CNTs embedded in three-parameter elastic medium. The governing torsional equation is obtained and solved for CNTs subjected to various boundary conditions and stated under different loads. The effects of different parameters on the angular displacement of the CNT are investigated. Sample angular displacements are tabulated and plotted for sensing the effect of all used parameters and to investigate the visco-Pasternak's parameters for future comparisons.

\section{Basic equation of carbon nanotube (CNT)}

Let us consider a single-walled carbon nanotube (SWCNT) of length $L$, inner radius $R_{1}$ and outer radius $R_{2}$ as shown in Figure 1. The CNT is made of a homogeneous isotropic and linearly elastic material with usual Lame's constants $\lambda$ and $\mu$ and the material density $\rho$.

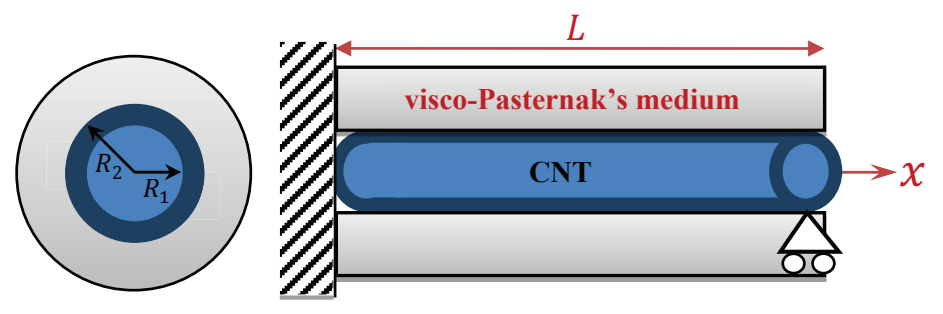

Figure 1. Schematic diagram for clamped-supported carbon nanotube embedded in visco-Pasternak's foundations.

The most general form of the constitutive relation in nonlocal elasticity theory involves an integral over the entire region of interest. The integral contains a nonlocal kernel function, which describes the relative influence of the strains at the various locations of the body on the stress at the material point under consideration. Specifically, the constitutive equation of nonlocal 
elasticity for homogenous and isotropic elastic solids read

$$
\sigma_{k l}(x)=\int_{\mathrm{v}} \alpha\left(\left|x-x^{\prime}\right|\right) \tau_{k l}\left(x^{\prime}\right) \mathrm{dv}\left(x^{\prime}\right),
$$

where $\sigma_{j}$ is the nonlocal stress tensor, $\mathrm{v}$ is the volume occupied by the elastic body, $\left|x-x^{\prime}\right|$ denotes distance in Euclidean space, and the nonlocal kernel $\alpha\left(\left|x-x^{\prime}\right|\right)$ accounts for the effect of the strain at the point $x^{\prime}$ on the stress at the point $x$ in the elastic body. The quantity $\tau_{k l}\left(x^{\prime}\right)$ denotes the local stress tensor for which the standard local constitutive equation is adopted, i.e.

$$
\tau_{k l}\left(x^{\prime}\right)=\lambda \varepsilon_{m m}\left(x^{\prime}\right) \delta_{k l}+2 \mu \varepsilon_{k l}\left(x^{\prime}\right),
$$

where $\varepsilon_{k l}\left(x^{\prime}\right)$ is the classical local strain tensor at $x^{\prime}$. The small straindisplacement relations are given by the usual relations

$$
\varepsilon_{k l}\left(x^{\prime}\right)=\frac{1}{2}\left(\frac{\partial v_{k}\left(x^{\prime}\right)}{\partial x_{l}^{\prime}}+\frac{\partial v_{l}\left(x^{\prime}\right)}{\partial x_{k}^{\prime}}\right),
$$

where $v_{l}\left(x^{\prime}\right)$ is the displacement vector at a reference point $x^{\prime}$ in the body. For an appropriate form of the nonlocal kernel [10], it turns out that the nonlocal internal constitutive relation given by Eq. (2.1) can be inverted to yield the following pseudo-local constitutive equation of gradient type

$$
\left[1-\left(a e_{0}\right)^{2} \nabla^{2}\right] \sigma_{k l}=\tau_{k l},
$$

where $\nabla^{2}$ is the Laplacian. The parameter $a$ is an internal characteristic length (e.g., lattice parameter, granular distance), and $e_{0}$ is a material constant determined by experiment or by matching dispersion curves of plane waves with those of atomic lattice dynamics. One may see that when the internal characteristic length $a$ is neglected, i.e. the particles of the medium are considered to be continuously distributed and interacting without long-range forces, $a e_{0}$ is zero, and Eq. (2.2) reduces to the constitutive equation of classical local thermoelasticity.

The present CNT is embedded in a homogeneous three-parameter viscoelastic medium. The foundation model is characterized by the linear Winkler's modulus $K_{1}$, the Pasternak's (shear) foundation modulus $K_{2}$, and the damping coefficient $c_{t}$ of the viscoelastic medium. Taking into account the unbonded contact between the CNT and medium, the interaction follows the three-parameter visco-Pasternak-type foundation model as

$$
R_{f}=\left(K_{1}-K_{2} L^{2} \frac{\partial^{2}}{\partial x^{2}}+c_{t} \frac{\partial}{\partial t}\right) v(x, t),
$$

where $v$ is the angular displacement of CNT and $R_{f}$ defines the external torque load and means elastic and damping effect of viscoelastic medium. Here, we have introduced the CNT length $L$ in Eq. (2.3) for maintaining the dimension of $K_{1}$ and $K_{2}$ to be the same. This model is simply reducing to the viscoWinkler's type when $K_{2}=0$. The viscosity term may be omitted by setting 
$c_{t}=0$ to get the analysis of the nanotube embedded in elastic medium. If the Newton's second law is applied to torsional vibration of the CNT, the governing equation is obtained as [6]

$$
\mu I_{p} \frac{\partial^{2} v}{\partial x^{2}}=\rho I_{p} \frac{\partial^{2} v}{\partial t^{2}}+R_{f}-q(x, t)
$$

where $q(x, t)$ is the external applied load and $I_{p}$ is the polar moment of inertia $I_{p}=\frac{\pi}{2}\left(R_{2}^{4}-R_{1}^{4}\right)$. In view of Eq. (2.2), the relevant nonlocal constitutive equation for the torsional deformation of the uniform CNT can be written in a one dimensional form

$$
\tau-\xi \frac{\partial^{2} \tau}{\partial t^{2}}=\mu \gamma
$$

where $\xi=\left(a e_{0}\right)^{2}$ is the nonlocal parameter, $\gamma$ is the shear strain and $\tau$ is the shear stress. The stress resultant $S$ due to the shear stress and torque relation $T$ are expressed as

$$
\{S, T\}=\int_{A} \tau\{1, z\} \mathrm{d} A,
$$

where $A$ is the cross-section of the CNT. Accordingly, the nonlocal stress resultant $S$ and torque relation $T$, with aid of Eq. (2.5), are given by the expressions

$$
S-\xi \frac{\partial^{2} S}{\partial x^{2}}=\mu A \gamma, \quad T-\xi \frac{\partial^{2} T}{\partial x^{2}}=\mu I_{p} \frac{\partial v}{\partial x} .
$$

If the above equation is inserted into Eq. (2.4), with the aid of Eq. (2.3), one can obtain the governing equation of motion for the CNT embedded in a visco-Pasternak's medium as

$$
\mu I_{p} \frac{\partial^{2} v}{\partial x^{2}}=\rho I_{p}\left(\frac{\partial^{2} v}{\partial t^{2}}-\xi \frac{\partial^{4} v}{\partial x^{2} \partial t^{2}}\right)+R_{f}-q(x, t)-\xi \frac{\partial^{2}}{\partial x^{2}}\left[R_{f}-q(x, t)\right] .
$$

\section{Solution of the problem}

With the harmonic vibration assumption, the angular displacement $v(x, t)$ of the CNT can be defined as

$$
\{v, q\}(x, t)=\{V(x), Q(x)\} \mathrm{e}^{\omega t},
$$

where $\omega$ represents the complex angular parameter for the CNT and $V(x)$ is the amplitude of the torsional displacement.

In what follows we will use the following dimensionless variables,

$$
x^{\prime}=\frac{x}{L}, \quad \xi^{\prime}=\frac{x}{L^{2}}, \quad \Omega=L \sqrt{\frac{\rho}{\mu}} \omega, \quad\left\{\kappa_{1}, \kappa_{2}\right\}=\frac{L^{2}}{\mu I_{p}}\left\{K_{1}, K_{2}\right\} .
$$

Then, the governing equation given in Eq. (2.6), after using Eq. (3.1) and the above dimensionless form become (dropping the dashed for convenience)

$$
\frac{\mathrm{d}^{4} V}{\mathrm{~d} x^{4}}-\bar{\alpha}_{0} \frac{\mathrm{d}^{2} V}{\mathrm{~d} x^{2}}+\beta_{0}^{2} V=\bar{q}(x)
$$


where

$$
\begin{aligned}
& \bar{\alpha}_{0}=\alpha_{0}+\xi \beta_{0}^{2}, \quad \alpha_{0}=\frac{1+\kappa_{2}}{\kappa_{2} \xi}, \\
& \beta_{0}^{2}=\frac{\Omega^{2}+\zeta \Omega+\kappa_{1}}{\kappa_{2} \xi}, \quad \bar{q}(x)=\frac{1}{\kappa_{2} \xi \mu I_{p}}\left(Q-\xi \frac{\mathrm{d}^{2} Q}{\mathrm{~d} x^{2}}\right),
\end{aligned}
$$

in which $\zeta=\frac{L}{\sqrt{\rho \mu} I_{p}} c_{t}, \Omega$ is the dimensionless frequency parameter, $\kappa_{1}$ is the dimensionless Winkler's foundation parameter, and $\kappa_{2}$ is the dimensionless Pasternak's foundation parameter. The general solution of Eq. (3.2) can be written as

$$
V(x)=c_{1} \mathrm{e}^{\eta_{1} x}+c_{2} \mathrm{e}^{-\eta_{1} x}+c_{3} \mathrm{e}^{\eta_{2} x}+c_{4} \mathrm{e}^{-\eta_{2} x}+p(x)
$$

where $c_{j}$ are undetermined integral constants, $p(x)$ is the particular solution of the non-homogeneous differential equation, and

$$
\eta_{1}=\frac{1}{\sqrt{2}} \sqrt{\bar{\alpha}_{0}-\sqrt{\bar{\alpha}_{0}^{2}-4 \beta_{0}^{2}}}, \quad \eta_{2}=\frac{1}{\sqrt{2}} \sqrt{\bar{\alpha}_{0}+\sqrt{\bar{\alpha}_{0}^{2}-4 \beta_{0}^{2}}} .
$$

The function $p(x)$ will be given according to the type of the applied load and the four constants $c_{j}$ may be determined by the application of the boundary conditions. The distribution of shear stress in the CNT must satisfy the conditions with respect to the angular displacement at the boundary. For the present $\mathrm{CNT}$, the solution given in Eq. (3.3) requires that two boundary conditions be satisfy at each edge. These may be a given angular displacement and slope, or force and moment, or some other combination. We can now formulate a variety of commonly encountered situations. The boundary conditions at the edges $x=0$ and $x=1$ of the CNT may readily be clamped, fixed, or built-in edge $(C)$ with $V=0$ and $\frac{\mathrm{d} V}{\mathrm{~d} x}=0$. For the simply-supported edge $(S)$ of the CNT we have $V=0$ and $\frac{\mathrm{d}^{2} V}{\mathrm{~d} x^{2}}=0$. So, one can discuss the following three cases of boundary conditions: $C-C, S-S$ and $C-S$.

\section{Frequency equation for different boundary conditions and external loads}

By applying the set of boundary conditions on the general solution given in Eq. (3.3) we get a set of algebraic equations in terms of the four constants $c_{j}$. These equations may be expressed in matrix form according to the boundary conditions used.

The CNT subjected to $C$ - $C$ boundary conditions:

$$
\left[\begin{array}{cccc}
1 & 1 & 1 & 1 \\
\mathrm{e}^{\eta_{1}} & \mathrm{e}^{-\eta_{1}} & \mathrm{e}^{\eta_{2}} & \mathrm{e}^{-\eta_{2}} \\
\eta_{1} & -\eta_{1} & \eta_{2} & -\eta_{2} \\
\eta_{1} \mathrm{e}^{\eta_{1}} & -\eta_{1} \mathrm{e}^{-\eta_{1}} & \eta_{2} \mathrm{e}^{\eta_{2}} & -\eta_{2} \mathrm{e}^{-\eta_{2}}
\end{array}\right]\left\{\begin{array}{l}
c_{1} \\
c_{2} \\
c_{3} \\
c_{4}
\end{array}\right\}=-\left\{\begin{array}{c}
p(0) \\
p(1) \\
p^{\prime}(0) \\
p^{\prime}(1)
\end{array}\right\} .
$$


The CNT subjected to $S$-S boundary conditions:

$$
\left[\begin{array}{cccc}
1 & 1 & 1 & 1 \\
\mathrm{e}^{\eta_{1}} & \mathrm{e}^{-\eta_{1}} & \mathrm{e}^{\eta_{2}} & \mathrm{e}^{-\eta_{2}} \\
\eta_{1}^{2} & \eta_{1}^{2} & \eta_{2}^{2} & \eta_{2}^{2} \\
\eta_{1}^{2} \mathrm{e}^{\eta_{1}} & \eta_{1}^{2} \mathrm{e}^{-\eta_{1}} & \eta_{2}^{2} \mathrm{e}^{\eta_{2}} & \eta_{2}^{2} \mathrm{e}^{-\eta_{2}}
\end{array}\right]\left\{\begin{array}{c}
c_{1} \\
c_{2} \\
c_{3} \\
c_{4}
\end{array}\right\}=-\left\{\begin{array}{c}
p(0) \\
p(1) \\
p^{\prime \prime}(0) \\
p^{\prime \prime}(1)
\end{array}\right\} .
$$

The CNT subjected to $C-S$ boundary conditions:

$$
\left[\begin{array}{cccc}
1 & 1 & 1 & 1 \\
\mathrm{e}^{\eta_{1}} & \mathrm{e}^{-\eta_{1}} & \mathrm{e}^{\eta_{2}} & \mathrm{e}^{-\eta_{2}} \\
\eta_{1} & -\eta_{1} & \eta_{2} & -\eta_{2} \\
\eta_{1}^{2} \mathrm{e}^{\eta_{1}} & \eta_{1}^{2} \mathrm{e}^{-\eta_{1}} & \eta_{2}^{2} \mathrm{e}^{\eta_{2}} & \eta_{2}^{2} \mathrm{e}^{-\eta_{2}}
\end{array}\right]\left\{\begin{array}{l}
c_{1} \\
c_{2} \\
c_{3} \\
c_{4}
\end{array}\right\}=-\left\{\begin{array}{c}
p(0) \\
p(1) \\
p^{\prime}(0) \\
p^{\prime \prime}(1)
\end{array}\right\} .
$$

In the above equations the prime means differentiation with respect to $x$. The different load forms of the external load are given by

$$
Q(x)=f(t)= \begin{cases}q_{0}, & \text { for uniform load (UL), } \\ q_{0} x, & \text { for linear load (LL), } \\ q_{0} \sin (\pi x) & \text { for sinusoidal load (SL), }\end{cases}
$$

where $q_{0}$ is the amplitude of the load at the center of the CNT. Using the above forms of the load, we can get the solution of Eqs. (4.1)-(4.2). The determination of the constants $c_{j}$ completes the full solution to the amplitude of the torsional displacement.

\section{$5 \quad$ Numerical results and discussions}

Let us consider some numerical examples to put into evidence the effect of the length $L$, the foundation parameters $\kappa_{1}$ and $\kappa_{2}$, and the viscous damping coefficient $c_{t}$ on torsional dynamic analysis of the present CNT. The dimensionless angular displacement parameter for the CNT embedded in a visco-Pasternak's medium is given as

$$
\bar{v}(x, t)=\frac{\mu}{h q_{0}} v(x, t)
$$

and it is carried out for different boundary conditions. The material properties for the present CNT are accepted as [6]

$$
\mu=460 \mathrm{GPa}, \quad \nu=0.19, \quad \rho=1.3 \mathrm{~g} / \mathrm{cm}^{3} .
$$

All plots are prepared by using the real value of the dimensionless angular displacement parameter $\bar{v}$. The CNT inner radius $R_{1}=0.68 \mathrm{~nm}$ and its thickness $h=R_{2}-R_{1}=0.08 \mathrm{~nm}$. The time is fixed at $t=0.3 \mathrm{~s}$ and the Pasternak's parameter is $\kappa_{2}=0.1 \mathrm{nN}$. The complex angular frequency is assumed as $\omega=\omega_{0}+i \varpi$ in which $\omega_{0}=1.25$ and $\varpi=0.25$. The computations are carried out (except otherwise stated) for $L=10 \mathrm{~nm}, \kappa_{1}=0.05 \mathrm{nN}, \xi=0.1$ $\mathrm{nm}^{2}$, and $c_{t}=0.5 \mathrm{nN}$. Other different values are given to the visco-Pasternak's 
parameters $c_{t}, \kappa_{1}$, and $\kappa_{2}$ and the length $L$ of the CNT. Here, we will discuss also the effect of the dimensionless nonlocal parameter $\xi$.

Benchmark results are presented in Table 1 for future comparisons with other investigators. The effects of the nonlocal parameter $\xi$, viscous damping coefficient $c_{t}$ and Winkler's parameter $\kappa_{1}$ on the angular displacement $\bar{v}$ of $C-C$ CNTs under different load with $L=11 \mathrm{~nm}$ are discussed. The CNT under sinusoidal load (SL) gives the highest angular displacement $\bar{v}$ while the CNT under linear load (LL) gives the smallest angular displacement $\bar{v}$. For a CNT subjected to any of the presented load, e.g., uniform load (UL), the angular displacement $\bar{v}$ is increasing with the increase of nonlocal parameter $\xi$. However, $\bar{v}$ is decreasing as the viscous damping coefficient $c_{t}$ and the Winkler's parameter $\kappa_{1}$ increase.

Table 1. Effect of the nonlocal parameter $\xi$, the viscous damping coefficient $c_{t}$ and the Winkler's parameter $\kappa_{1}$ on the angular displacement $\bar{v}$ of $C$ - $C$ CNTs under different load $(L=11 \mathrm{~nm})$.

\begin{tabular}{|c|c|c|c|c|c|c|c|c|c|c|}
\hline \multirow[b]{2}{*}{$c_{t}$} & \multirow[b]{2}{*}{$\kappa_{1}$} & \multicolumn{3}{|c|}{$\xi=0.1$} & \multicolumn{3}{|c|}{$\xi=0.3$} & \multicolumn{3}{|c|}{$\xi=0.5$} \\
\hline & & $\mathrm{UL}$ & $\mathrm{LL}$ & SL & $\mathrm{UL}$ & $\mathrm{LL}$ & SL & $\mathrm{UL}$ & $\mathrm{LL}$ & SL \\
\hline \multirow[t]{4}{*}{0.1} & 0.0 & 1.519 & 0.760 & 2.658 & 2.683 & 1.341 & 9.023 & 2.465 & 1.232 & 12.379 \\
\hline & 0.1 & 1.237 & 0.619 & 2.179 & 2.330 & 1.165 & 7.847 & 2.243 & 1.121 & 11.271 \\
\hline & 0.2 & 1.023 & 0.511 & 1.813 & 2.036 & 1.018 & 6.868 & 2.044 & 1.022 & 10.277 \\
\hline & 0.3 & 0.857 & 0.429 & 1.529 & 1.789 & 0.895 & 6.048 & 1.865 & 0.933 & 9.387 \\
\hline \multirow[t]{4}{*}{0.3} & 0.0 & 0.741 & 0.370 & 1.346 & 1.736 & 0.868 & 5.885 & 1.886 & 0.943 & 9.503 \\
\hline & 0.1 & 0.637 & 0.319 & 1.163 & 1.531 & 0.765 & 5.201 & 1.717 & 0.858 & 8.655 \\
\hline & 0.2 & 0.552 & 0.276 & 1.013 & 1.358 & 0.679 & 4.625 & 1.567 & 0.783 & 7.907 \\
\hline & 0.3 & 0.483 & 0.241 & 0.889 & 1.212 & 0.606 & 4.136 & 1.434 & 0.717 & 7.243 \\
\hline \multirow[t]{4}{*}{0.5} & 0.0 & 0.426 & 0.213 & 0.800 & 1.156 & 0.578 & 3.960 & 1.422 & 0.711 & 7.193 \\
\hline & 0.1 & 0.379 & 0.190 & 0.713 & 1.038 & 0.519 & 3.564 & 1.302 & 0.651 & 6.594 \\
\hline & 0.2 & 0.339 & 0.170 & 0.639 & 0.936 & 0.468 & 3.223 & 1.196 & 0.598 & 6.063 \\
\hline & 0.3 & 0.305 & 0.152 & 0.575 & 0.848 & 0.424 & 2.927 & 1.106 & 0.551 & 5.592 \\
\hline \multirow[t]{4}{*}{1.0} & 0.0 & 0.163 & 0.082 & 0.319 & 0.503 & 0.252 & 1.780 & 0.737 & 0.369 & 3.783 \\
\hline & 0.1 & 0.152 & 0.076 & 0.296 & 0.467 & 0.233 & 1.654 & 0.689 & 0.344 & 3.537 \\
\hline & 0.2 & 0.141 & 0.071 & 0.276 & 0.434 & 0.217 & 1.541 & 0.644 & 0.322 & 3.313 \\
\hline & 0.3 & 0.132 & 0.066 & 0.257 & 0.404 & 0.202 & 1.439 & 0.604 & 0.302 & 3.110 \\
\hline
\end{tabular}

Figures 2-4 show the effect of the nonlocal parameter $\xi$ on the variation of the angular displacement $\bar{v}$ along the axial direction of CNTs subjected to various boundary conditions and under different loads. The angular displacement $\bar{v}$ increases as $\xi$ increases for different cases. It is very sensitive to the variation of the load presented. The angular displacement of a CNT under uniform load is intermediate those of CNTs under linear and sinusoidal loads. The maximum angular displacement $\bar{v}$ occurs at the center of the $C-C$ and $S-S$ CNTs $(x=0.5)$ under uniform (Figure 2a and Figure 3a) and sinusoidal (Figure 2c and Figure 3c) loads. However, the maximum angular displacement $\bar{v}$ occurs near the center of the $C$-S CNT $(x \cong 0.555)$ under uniform (Figure 4a and Figure 4c) and sinusoidal loads. This is not the same for CNTs under linear load and subjected to various boundary conditions (Figure 2b, Figure 3b and 
Figure 4b).

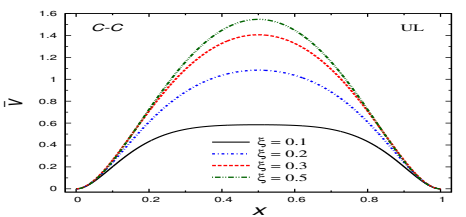

(a)

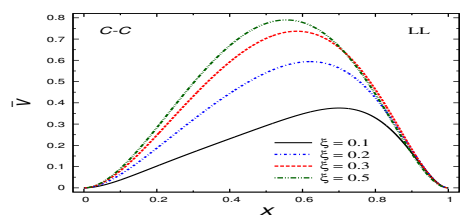

(b)

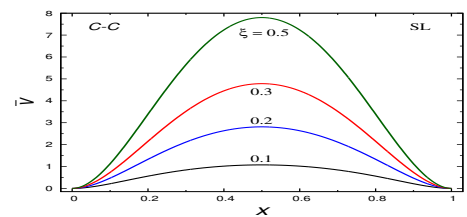

(c)

Figure 2. Effect of nonlocal parameter $\xi$ on the distribution of angular displacement along the axial direction of a C-C CNT subjected to: (a) UL, (b) LL, and (c) SL.

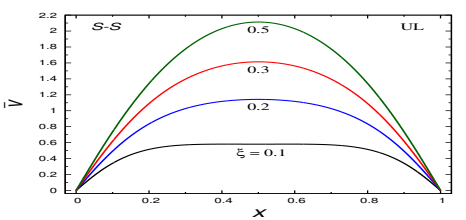

(a)

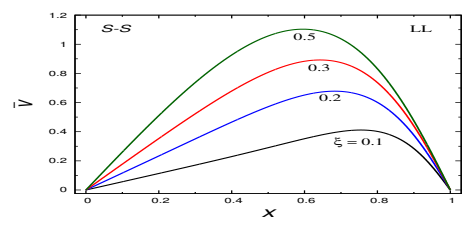

(b)

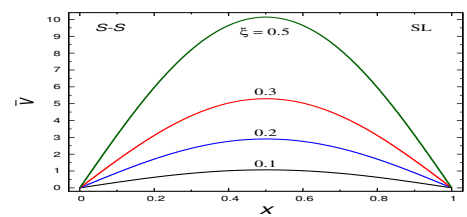

(c)

Figure 3. Effect of nonlocal parameter $\xi$ on the distribution of angular displacement along the axial direction of a $S-S$ CNT subjected to: (a) UL, (b) LL, and (c) SL.

Figures 5-7 show the effect of damping parameter $c_{t}$ on the variation of the angular displacement $\bar{v}$ along the axial direction of CNTs subjected to various boundary conditions and under different loads. The angular displacement $\bar{v}$ decreases as $c_{t}$ increases for different cases and it is very sensitive to the variation of the load presented. 


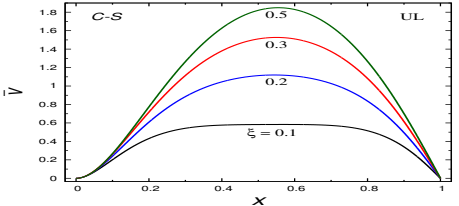

(a)

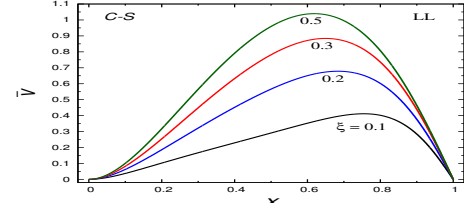

(b)

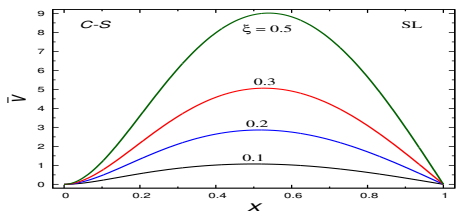

(c)

Figure 4. Effect of nonlocal parameter $\xi$ on the distribution of angular displacement along the axial direction of a $C$-S CNT subjected to: (a) UL, (b) LL, and (c) SL.

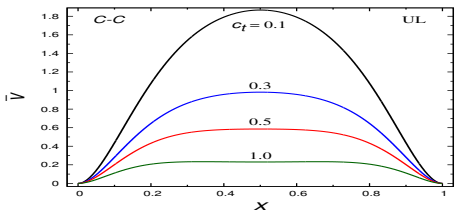

(a)

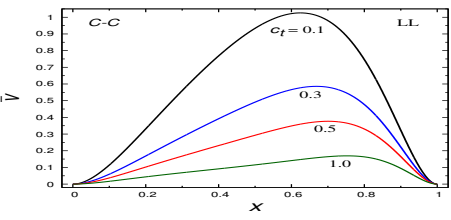

(b)

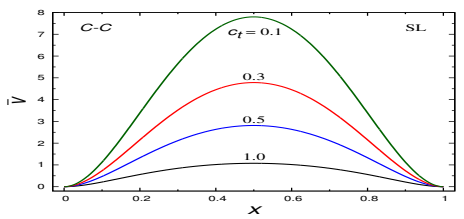

(c)

Figure 5. Effect of viscous damping parameter $c_{t}$ on the distribution of angular displacement along the axial direction of a $C$ - $C$ CNT subjected to: (a) UL, (b) LL, and (c) SL.

The maximum angular displacement $\bar{v}$ occurs at the center of the $C$ - $C$ and $S$ - $S$ CNTs under uniform (Figure 5a and Figure 6a) and sinusoidal (Figure 5c and Figure 6c) loads. However, the maximum angular displacement $\bar{v}$ occurs near the center of the $C$-S CNT under uniform (Figure 7a) and sinusoidal (Figure 7c) loads. This is not the same for CNTs under linear load and subjected to various boundary conditions (Figure 5b, Figure 6b and Figure $7 \mathrm{~b}$ ). For example, for a $C$ - $C$ CNT subjected to the linear load (Figure 5b), the 
maximum values $\bar{v}_{\max }=1.02608,0.58585,0.37654$, and 0.16936 which occur at $x=0.624,0.668,0.701$, and 0.752 for $c_{t}=0.1,0.3,0.5$ and 1.0 , respectively.

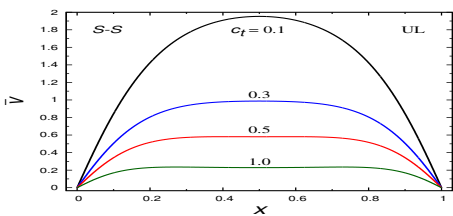

(a)

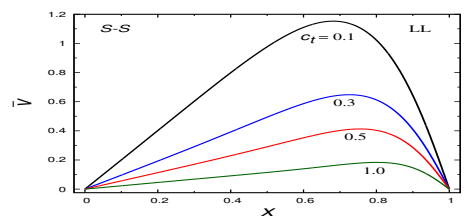

(b)

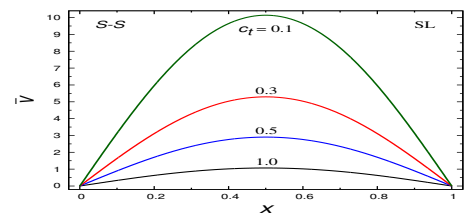

(c)

Figure 6. Effect of viscous damping parameter $c_{t}$ on the distribution of angular displacement along the axial direction of a $S$-S CNT subjected to: (a) UL, (b) LL, and (c) SL.

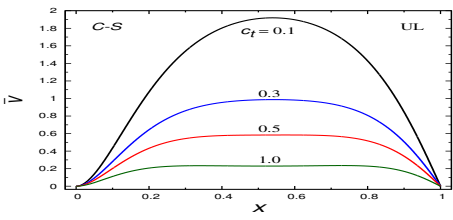

(a)

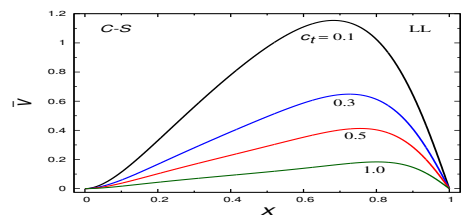

(b)

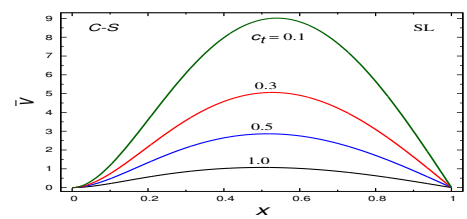

(c)

Figure 7. Effect of viscous damping parameter $c_{t}$ on the distribution of angular displacement along the axial direction of a $C-S$ CNT subjected to: (a) UL, (b) LL, and (c) SL.

Figures 8-10 show the effect of Winkler's parameter $\kappa_{1}$ on the variation of the angular displacement $\bar{v}$ along the axial direction of CNTs subjected to various boundary conditions and under different loads. The angular displacement 
$\bar{v}$ decreases as $\kappa_{1}$ increases for different cases and it is very sensitive to the variation of the load presented.

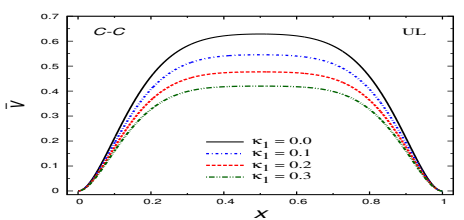

(a)

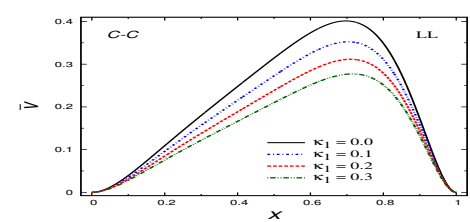

(b)

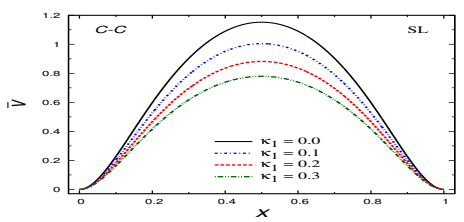

(c)

Figure 8. Effect of Winkler's parameter $\kappa_{1}$ on of the distribution of angular displacement along the axial direction of a $C$ - $C$ CNT subjected to: (a) UL, (b) LL, and (c) SL.

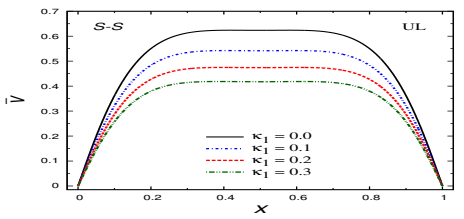

(a)

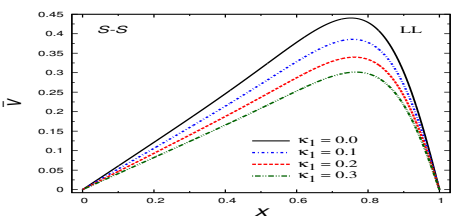

(b)

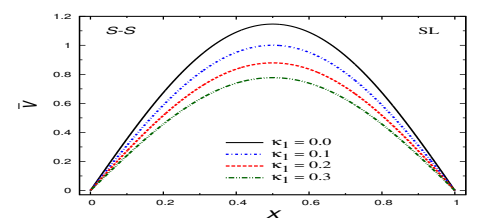

(c)

Figure 9. Effect of Winkler's parameter $\kappa_{1}$ on of the distribution of angular displacement along the axial direction of a $C$-S CNT subjected to: (a) UL, (b) LL, and (c) SL.

Once again, the maximum angular displacement $\bar{v}$ may be occur at the center of the CNTs under sinusoidal load only (Figure 8c, Figure 9c and Figure 10c). However, the maximum angular displacement $\bar{v}$ may be occur along the interval $(0.35<x<0.65)$ of the CNTs under uniform load only (Fig- 
ure 8a, Figure 9a and Figure 10a). This is not the same for CNTs under linear load and subjected to various boundary conditions (Figure 8b, Figure $9 \mathrm{~b}$ and Figure 10b).

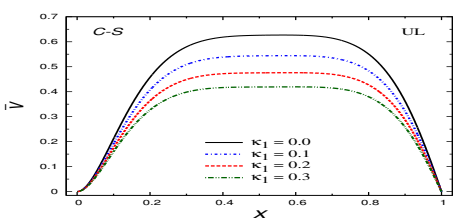

(a)

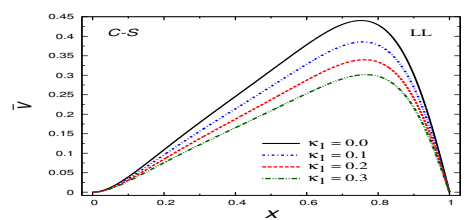

(b)

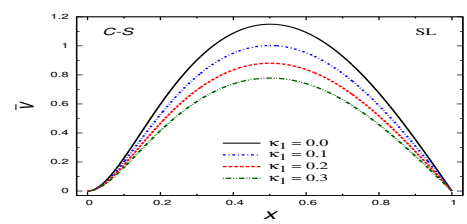

(c)

Figure 10. Effect of Winkler's parameter $\kappa_{1}$ on of the distribution of angular displacement along the axial direction of a $C$-S CNT subjected to: (a) UL, (b) LL, and (c) SL.

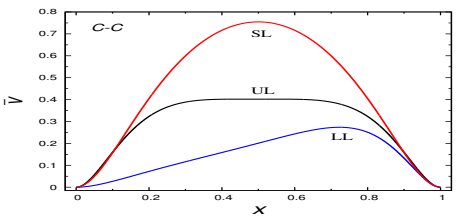

(a)

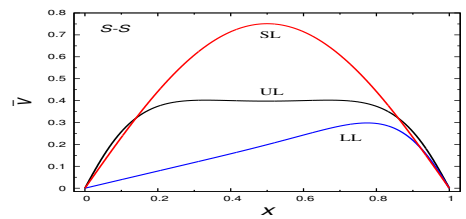

(b)

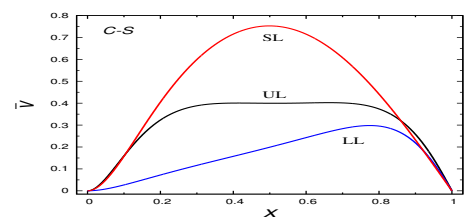

(c)

Figure 11. Effect of different loads on the distribution of angular displacement along the axial direction of a CNT subjected to: (a) $C-C$, (b) $S$ - $S$, and (c) $C-S$ boundary conditions.

It is interesting to note that there are two absolute maximum points have the same value for CNTs under uniform load for small values of $\xi$, greater values of $c_{t}$, and all values of $\kappa_{1}$. Let us consider the $S$-S CNT under uniform 
load as an example. For $\xi=0.1, \bar{v}_{\max }=0.58110$ at the two positions $x=0.412$ and $x=0.588$ (Figure 3a). For $c_{t}=1.0, \bar{v}_{\max }=0.23546$ at the two positions $x=0.271$ and $x=0.729$ (Figure $6 \mathrm{a}$ ). For $\kappa_{1}=0.3, \bar{v}_{\max }=0.41849$ at the two positions $x=0.385$ and $x=0.615$ (Figure $9 \mathrm{a}$ ).

Finally, Figure 11 shows the variation of the angular displacement along the axial direction of CNTs with $L=11 \mathrm{~nm}$ and subjected to various boundary conditions and different loads. The maximum angular displacement $\bar{v}$ occurs at the center of the CNTs under sinusoidal load irrespective of the boundary condition. As we discussed before, there are two absolute maximum points have the same value for CNTs under uniform load. If the applied load is linear, we have different positions for different maximum values of the angular displacement. For example, $\bar{v}_{\max }=0.27359$ at $x=0.723$ for the $C$ - $C$ CNT (Figure 11a); $\bar{v}_{\max }=0.29798$ at $x=0.774$ for the $S$ - $S$ CNT (Figure 11b); and $\bar{v}_{\max }=0.29797$ at $x=0.774$ for the $C-S \mathrm{CNT}$ (Figure 11c).

\section{Validation}

To the best of the author's knowledge no published literature is available for comparison the torsional dynamic response of the SWCNTs. In this article, we restrict our attention to investigate the effects of some parameters like nonlocal parameter, nanotube length, Winkler's modulus, Pasternak's shear foundation, and damping coefficient on the angular displacement of the nanotube. However, the present results can be validated by the other published literatures in the torsional statics and dynamics of SWCNTs just embedded in an elastic medium. In this regard, the simplified result of this paper is compared with the work of Arda and Aydogdu [6]. If we neglect the external applied load $q(x, y)$ and reduce the foundation model to be a Winkler's one, Eq. (2.6) should be tends to

$$
\mu I_{p} \frac{\partial^{2} v}{\partial x^{2}}=\rho I_{p}\left(\frac{\partial^{2} v}{\partial t^{2}}-\xi \frac{\partial^{4} v}{\partial x^{2} \partial t^{2}}\right)+K_{1}\left(v-\xi \frac{\partial^{2} v}{\partial x^{2}}\right),
$$

which indicates the governing equation of motion for the CNT embedded in elastic medium. As can be observed Eq. (6.1) is almost the same as that in Ref. [6]. In fact, the plots of the non-dimensional angular displacement of CNT presented in Ref [6] are considered as special cases when compared with the present results.

\section{Conclusions}

In this article, a carbon nanotube is embedded in a three-parameter viscoelastic foundation medium. The torsional dynamics analysis is performed due to the nonlocal elasticity theory. For the sake of completeness and comparisons, some angular displacements are tabulated here for different viscous damping coefficient, nonlocal parameter, and Winkler's elastic foundation. Different loads and various boundary conditions are taken into consideration. The angular displacement is very sensitive to the variation of the load presented. The angular displacement of a CNT under uniform load is intermediate those of 
CNTs under linear and sinusoidal loads. The maximum angular displacement $\bar{v}$ occurs at the center of the $C-C$ and $S$-S CNTs under uniform and sinusoidal loads. However, the maximum angular displacement occurs near the center of the $C$-S CNT under uniform and sinusoidal loads. This is not the same for CNTs under linear loads and subjected to various boundary conditions. This investigation may give a useful help in applications of nanotubes technology, especially those that embedded in viscoelastic medium.

\section{References}

[1] P.M. Ajayan and O.Z. Zhou. Applications of carbon nanotubes. Topics Appl. Phys., 80:391-425, 2001. http://dx.doi.org/10.1007/3-540-39947-X_14.

[2] R. Ansari, R. Gholami and S. Ajori. Torsional vibration analysis of carbon nanotubes based on the strain gradient theory and molecular dynamic simulations. J. Vib. Acoust., 135(5):051016, 2013. http://dx.doi.org/10.1115/1.4024208.

[3] A.G. Arani, M.R. Bagheri, R. Kolahchi and Z.K. Maraghi. Nonlinear vibration and instability of fluid-conveying DWBNNT embedded in a visco-Pasternak medium using modified couple stress theory. J. Mech. Sci. Tech., 27:2645-2658, 2013. http://dx.doi.org/10.1007/s12206-013-0709-3.

[4] A.G. Arani and M.A. Roudbari. Nonlocal piezoelastic surface effect on the vibration of visco-Pasternak coupled boron nitride nanotube system under a moving nanoparticle. Thin Solid Films, 542:232-241, 2013. http://dx.doi.org/10.1016/j.tsf.2013.06.025.

[5] A.G. Arani, M.S. Zarei, M. Mohammadimehr, A. Arefmanesh and M.R. Mozdianfard. The thermal effect on buckling analysis of a DWCNT embedded on the Pasternak foundation. Phys. E, 43(9):1642-1648, 2011. http://dx.doi.org/10.1016/j.physe.2011.05.014.

[6] M. Arda and M. Aydogdu. Torsional statics and dynamics of nanotubes embedded in an elastic medium. Compos. Struct., 114:80-91, 2014. http://dx.doi.org/10.1016/j.compstruct.2014.03.053.

[7] R.C. Batra and A. Sears. Continuum models of multi-walled carbon nanotubes. Int. J. Solids Struct., 44(22-23):7577-7596, 2007. http://dx.doi.org/10.1016/j.ijsolstr.2007.04.029.

[8] A.C. Eringen. Nonlocal polar elastic continua. Int. J. Eng. Sci., 10:1-16, 1972.

[9] A.C. Eringen. On differential equations of nonlocal elasticity and solutions of screw dislocation and surface waves. J. Appl. Phys., 54:4703-4710, 1983. http://dx.doi.org/10.1063/1.332803.

[10] A.C. Eringen. Nonlocal Continuum Field Theories. New York: Springer Verlag, 2002.

[11] R.F. Gibson, E.O. Ayorinde and Y-F. Wen. Vibrations of carbon nanotubes and their composites: a review. Compos. Sci. Tech., 67(1):1-28, 2007. http://dx.doi.org/10.1016/j.compscitech.2006.03.031.

[12] S. Govindjee and J.L. Sackman. On the use of continuum mechanics to estimate the properties of nanotubes. Solid State Commun., 110(4):227-230, 1999. http://dx.doi.org/10.1016/S0038-1098(98)00626-7. 
[13] I.A. Guz, A.A. Rodger, A.N. Guz and J.J. Rushchitsky. Developing the mechanical models for nanomaterials. Compos. A, 38(4):1234-1250, 2007. http://dx.doi.org/10.1016/j.compositesa.2006.04.012.

[14] Q. Han and G. Lu. Torsional buckling of a double-walled carbon nanotube embedded in an elastic medium. Eur. J. Mech. A/solids, 22(6):875-883, 2003. http://dx.doi.org/10.1016/j.euromechsol.2003.07.001.

[15] M.J. Hao, X.M. Guo and Q. Wang. Small-scale effect on torsional buckling of multiwalled carbon nanotubes. Eur. J. Mech. A/Solids, 29(1):49-55, 2010. http://dx.doi.org/10.1016/j.euromechsol.2009.05.008.

[16] S. Iijima. Helical microtubes of graphitic carbon. Nature, 354:56-58, 1991.

[17] F. Khademolhosseini, A.S. Phani, A. Nojeh and N. Rajapakse. Nonlocal continuum modeling and molecular dynamics simulation of torsional vibration of carbon nanotubes. IEEE Transact. Nanotechn., 11(1):34-43, 2012. http://dx.doi.org/10.1109/TNANO.2011.2111380.

[18] M. Mohammadimehr, A.R. Saidi, A.G. Arani, A. Arefmanesh and Q. Han. Torsional buckling of a DWCNT embedded on Winkler and Pasternak foundations using nonlocal theory. J. Mech. Sci. Tech., 24(6):1289-1299, 2010. http://dx.doi.org/10.1007/s12206-010-0331-6.

[19] T. Murmu, S. Adhikari and C.Y. Wang. Torsional vibration of carbon nanotubebuckyball systems based on nonlocal elasticity theory. Phys. E, 43(6):1276-1280, 2011. http://dx.doi.org/10.1016/j.physe.2011.02.017.

[20] T. Murmu and S.C. Pardhan. Buckling analysis of a single-walled carbon nanotube embedded in an elastic medium based on nonlocal elasticity and Timoshenko beam theory and using DQM. Phys. E, 41(7):1232-1239, 2009. http://dx.doi.org/10.1016/j.physe.2009.02.004.

[21] M.B. Nardelli, C. Brabec and A. Maiti. Lip-lip interactions and the growth of multiwalled carbon nanotubes. Phys. Rev. Lett., 80(2):313-316, 1998. http://dx.doi.org/10.1103/PhysRevLett.80.313.

[22] T. Natsuki, T. Tsuchiya, Q.Q. Ni and M. Endo. Torsional elastic instability of double walled carbon nanotubes. Carbon, 48(15):4362-4368, 2010. http://dx.doi.org/10.1016/j.carbon.2010.07.050.

[23] D. Qian, G.J. Wagner, W.K. Liu, M.F. Yu and R.S. Ruoff. Mechanics of carbon nanotubes. Appl. Mech. Rev., 55(6):495-533, 2002. http://dx.doi.org/10.1115/1.1490129.

[24] P. Soltani, M.M. Taherian and A. Farshidianfar. Vibration and instability of a viscous-fluid-conveying single-walled carbon nanotube embedded in a visco-elastic medium. J. Phys. D: Appl. Phys., 43(42):425401, 2010. http://dx.doi.org/10.1088/0022-3727/43/42/425401.

[25] C. Sun and K. Liu. Combined torsional buckling of multi-walled carbon nanotubes coupling with axial loading and radial pressures. Int. J. Solids Struct., 45(7-8):2128-2139, 2008. http://dx.doi.org/10.1016/j.ijsolstr.2007.11.009.

[26] X. Wang, G.X. Lu and Y.J. Lu. Buckling of embedded multi-walled carbon nanotubes under combined torsion and axial loading. Int. J. Solids Struct., 44(1):336-351, 2007. http://dx.doi.org/10.1016/j.ijsolstr.2006.04.031.

[27] H.K. Yang and X. Wang. Bending stability of multi-wall carbon nanotubes embedded in an elastic medium. Model. Simulat. Mater. Sci. Eng., 14(1):99116, 2006. http://dx.doi.org/10.1088/0965-0393/14/1/008. 
[28] H.K. Yang and X. Wang. Torsional buckling of multi-wall carbon nanotubes embedded in an elastic medium. Compos. Struct., 77(2):182-189, 2007. http://dx.doi.org/10.1016/j.compstruct.2005.06.013.

[29] J. Yoon, C.Q. Ru and A. Mioduchowski. Vibration of an embedded multiwall carbon nanotube. Compos. Sci. Tech., 63(11):1533-1542, 2003. http://dx.doi.org/10.1016/S0266-3538(03)00058-7.

[30] W. Yu, N. Xiang-Gui, W. Xiu-Xi and W. Heng-An. Effect of temperature on deformation of carbon nanotube under compression. Chinese Phys., 12(9):10071010, 2003. http://dx.doi.org/10.1088/1009-1963/12/9/315.

[31] A.M. Zenkour. Nonlocal transient thermal analysis of a single-layered graphene sheet embedded in viscoelastic medium. Phys. E, 79:87-97, 2016. http://dx.doi.org/10.1016/j.physe.2015.12.003.

[32] A.M. Zenkour. Vibration analysis of a single-layered graphene sheet embedded in visco-Pasternak's medium using nonlocal elasticity theory. J. Vibroeng., 18(4):2319-2330, 2016. http://dx.doi.org/10.21595/jve.2016.16585.

[33] Y.-X. Zhen, B. Fang and Y. Tang. Thermal-mechanical vibration and instability analysis of fluid-conveying double walled carbon nanotubes embedded in visco-elastic medium. Phys. E, 44(2):379-385, 2011. http://dx.doi.org/10.1016/j.physe.2011.09.004. 